\title{
Commutative feebly nil-clean group rings
}

\author{
Peter V. Danchev \\ Institute of Mathematics and Informatics, \\ Bulgarian Academy of Sciences, Sofia, Bulgaria \\ email: danchev@math.bas.bg; \\ pvdanchev@yahoo.com
}

\begin{abstract}
An arbitrary unital ring $\mathrm{R}$ is called feebly nil-clean if any its element is of the form $q+e-f$, where $q$ is a nilpotent and $e, f$ are idempotents with ef $=f e$. For any commutative ring $R$ and any abelian group G, we find a necessary and sufficient condition when the group $\operatorname{ring} R(G)$ is feebly nil-clean only in terms of $R, G$ and their sections. Our result refines establishments due to McGovern et al. in J. Algebra Appl. (2015) on nil-clean rings and Danchev-McGovern in J. Algebra (2015) on weakly nil-clean rings, respectively.
\end{abstract}

\section{Introduction and background}

Throughout the text of this short paper, all rings $\mathrm{R}$ are assumed to be associative and commutative, containing identity element which differs from the zero element. Our terminology and notations are mainly in agreement with [10] and [11]. For instance, $J(R)$ denotes the Jacobson radical of $R$, and $N(R)$ denotes the nil-radical of $R$. Also, let everywhere in the text $G$ be a multiplicative abelian group, and let $R(G)$ be the group ring of $G$ over R. As usual, $G_{p}$ stands for the $p$-torsion component of the group $G$ with p-socle $G[p]=\left\{a \in G \mid a^{p}=1\right\}$, and we shall say that the group $G$ is a p-group, provided $G=G_{p}$. Likewise, we set $G^{p}=\left\{g^{\mathfrak{p}} \mid g \in G\right\}$ to be the p-th power subgroup of the group $G$.

A ring $R$ is known to be nil-clean if, for every $r \in R$, there are a nilpotent $\mathrm{q} \in \mathrm{R}$ and an idempotent $\mathrm{e} \in \mathrm{R}$ such that $\mathrm{r}=\mathrm{q}+e$. The next necessary and

2010 Mathematics Subject Classification: 16D60; 16S34; 16U60

Key words and phrases: nil-clean rings, weakly nil-clean rings, feebly nil-clean rings, group rings 
sufficient condition for a commutative group ring to be nil-clean was recently obtained in [9]. Specifically, the following holds: The commutative group ring $\mathrm{R}(\mathrm{G})$ is nil-clean if, and only if, the ring $\mathrm{R}$ is nil-clean and the group $\mathrm{G}$ is a 2-group.

Generalizing this, a ring $\mathrm{R}$ is known to be weakly nil-clean if, for every $r \in R$, there are a nilpotent $q \in R$ and an idempotent $e \in R$ such that $r=q+e$ or $r=q-e$. Alternatively, a necessary and sufficient condition for a commutative group ring to be weakly nil-clean was recently obtained in [4]. Precisely, the following holds: The commutative group ring $\mathrm{R}(\mathrm{G})$ is weakly nil-clean if, and only if, either $\mathrm{G}=\{1\}$ and $\mathrm{R}$ is weakly nil-clean, or $\mathrm{R}$ is nil-clean and $\mathrm{G}$ is a non-trivial 2-group, or $\mathrm{R} / \mathrm{N}(\mathrm{R}) \cong \mathbb{Z}_{3}$ and $\mathrm{G}$ is a non-trivial 3-group.

As a common generalization of these two definitions for nil-clean and weakly nil-clean rings, a ring $\mathrm{R}$ is known to be feebly nil-clean (see, for example, [1] and [2]) if, for every $r \in R$, there are a nilpotent $q \in R$ and two idempotents $e, f \in R$ such that $r=q+e-f$. So, the leitmotif of writing up this brief article is to generalize somewhat the above two claims by obtaining a criterion for an arbitrary commutative group ring to be feebly nil-clean.

For completeness of the exposition, it is worthwhile noticing that an extension of the aforementioned nil-clean rings are the so-called $U U$ rings that are rings whose units are unipotents (i.e., the sum of 1 and some nilpotent). In [3] were examined commutative UU group rings. Exactly, it was proved in Corollary 2.3 there that $\mathrm{R}(\mathrm{G})$ is a commutative $U U$ ring if, and only if, $\mathrm{R}$ is a commutative $U U$ ring and $\mathrm{G}$ is an abelian 2-group.

\section{Main results}

Before proving our chief statement, we need the following two key formulas from [7] and [8], respectively. In fact, appealing to [7], one writes the formula

$$
J(R(G))=J(R)(G)+\left\langle r(g-1) \mid g \in G_{p}, p r \in J(R)\right\rangle .
$$

In that aspect, consulting with [8], one writes the formula

$$
\mathrm{N}(\mathrm{R}(\mathrm{G}))=\mathrm{N}(\mathrm{R})(\mathrm{G})+\left\langle\mathrm{r}(\mathrm{g}-1) \mid \mathrm{g} \in \mathrm{G}_{\mathrm{p}}, \mathrm{pr} \in \mathrm{N}(\mathrm{R})\right\rangle .
$$

Standardly, I(R(G); G) designates the fundamental (augmentation) ideal of $R(G)$ with respect to $G$ with basis consisting of all elements of the type $1-g$, where $\mathrm{g} \in \mathrm{G}$. It is well known that the isomorphisms

$$
R(G) /[N(R)(G)+I(R(G) ; G)] \cong R / N(R)
$$


and

$$
\mathrm{R}(\mathrm{G}) / \mathrm{I}(\mathrm{R}(\mathrm{G}) ; \mathrm{G}) \cong \mathrm{R}
$$

are valid. We will now prove something similar and helpful for us for further use.

Proposition 1 Let $\mathrm{R}$ be a ring and $\mathrm{G}$ a group. Then the following isomorphism is fulfilled:

$$
R(G) / N(R)(G) \cong(R / N(R))(G) .
$$

Proof. There is the natural ring surjection $R \rightarrow R / N(R)$ which induces by the usual element-wise manipulation the ring surjective homomorphism $R(G) \rightarrow$ $(R / N(R))(G)$. This epimorphism obviously has kernel $N(R) G$ and henceforth the well-known Homomorphism Theorem applies to get the desired assertion.

A ring is boolean if each its element is an idempotent. Let us recall that a ring is said to be tripotent if each its element $x$ satisfies the equation $x^{3}=x$. These rings are necessarily commutative being also a subdirect product of a family of single or isomorphic copies of the fields $\mathbb{Z}_{2}$ and $\mathbb{Z}_{3}$ (see, e.g., [6]). Likewise, as $6=0$ here, any tripotent ring $R$ can be decomposed as the direct product of two rings $R_{1} \times R_{2}$, where $R_{1}$ is boolean and $R_{2}$ is tripotent of characteristic 3 . It is pretty evident that reduced feebly nil-clean rings are themselves tripotent.

We begin our work with a few useful technicalities.

Lemma 1 The next two statements are true:

(i) A direct factor of a feebly nil-clean ring is a feebly nil-clean ring as well.

(ii) The direct product of two feebly nil-clean rings is also a feebly nil-clean ring.

Proof. Straightforward by a direct check, so that we leave it to the interested reader.

Lemma 2 An epimorphic image of a feebly nil-clean ring is too a feebly nilclean ring.

Proof. Since nilpotents and idempotents map under any homomorphism again into nilpotents and idempotents, respectively, the claim follows elementarily.

Proposition 2 Suppose that $\mathrm{R}$ is a commutative ring. Then the following three points are equivalent: 
(i) $\mathrm{R}$ is feebly nil-clean.

(ii) $\mathrm{J}(\mathrm{R})$ is nil and $\mathrm{R} / \mathrm{J}(\mathrm{R})$ is tripotent.

(iii) $\mathrm{R} / \mathrm{N}(\mathrm{R})$ is tripotent.

Proof. It suffices to prove only the equivalence (i) $\Longleftrightarrow$ (iii), because whenever $J(R)$ is nil we will have that $J(R)=N(R)$ as well as, in accordance with [1] or [2], $R$ being feebly nil-clean yields that $J(R)$ is nil. To this purpose, the implication (i) $\Rightarrow$ (iii) follows at once by the usage of Lemma 2 .

As for the converse implication (i) $\Leftarrow$ (iii), we may write by consulting with $[6$, Theorem 1] accomplished with a simple trick that every element of $R / N(R)$ is the difference of two idempotents, say $r+N(R)=\left(e_{1}+N(R)\right)-\left(e_{2}+N(R)\right)=$ $e_{1}-e_{2}+N(R)$, where $r \in R$ is an arbitrary element and $e_{1}, e_{2} \in R$ are some elements. But as it is well-known as a folklore fact, we may choose these $e_{1}$ and $e_{2}$ to be idempotents. Consequently, one follows that $r=t+e_{1}-e_{2}$ for some nilpotent $t$ in $R$, as expected.

As an immediate consequence, one yields the following.

Corollary 1 Let $\mathrm{I}$ be a nil ideal of a ring $\mathrm{R}$. Then $\mathrm{R}$ is feebly nil-clean if, and only if, R/I is feebly nil-clean.

Proof. The "necessity" follows by virtue of Lemma 2. As for the "sufficiency", because of the inclusion $I \subseteq N(R)$, there exists an epimorphism $R / I \rightarrow R / N(R)$ with kernel $N(R) / I=N(R / I)$. Hence $R / N(R)$ is feebly nil-clean, i.e., tripotent. Furthermore, we apply Proposition 2 to conclude the claim.

We now have all the ingredients necessary to proceed by proving the following chief assertion, which gives a necessary and sufficient condition when a commutative group ring will be feebly nil-clean.

Theorem 1 Suppose $\mathrm{R}$ is a commutative ring and $\mathrm{G}$ is an abelian group. Then the group ring $\mathrm{R}(\mathrm{G})$ is feebly nil-clean if, and only if, exactly one of the following three items is valid:

(1) $\mathrm{G}=\{1\}$ and $\mathrm{R}$ is feebly nil-clean.

(2) $\mathrm{G} \neq\{1\}$ and $\mathrm{R} / \mathrm{N}(\mathrm{R}) \cong \mathrm{R}_{1} \times \mathrm{R}_{2}$, where $\mathrm{R}_{1}$ is boolean and $\mathrm{R}_{2}$ is tripotent of characteristic 3 such that

(a) $\mathrm{R}_{1}=\{0\}$, or $\mathrm{R}_{1} \neq\{0\}$ and $\mathrm{G}$ is a 2-group;

(b) $\mathrm{R}_{2}=\{0\}$, or $\mathrm{R}_{2} \neq\{0\}$ and either $\mathrm{G}=\mathrm{G}_{3}$ or $\mathrm{G}=\mathrm{G}_{3} \times \mathrm{G}$ [2].

Proof. "Left-to-right". The assumption that G is the trivial group leads to $R(G) \cong R$, so that we may assume without loss of generality that $G$ is non-trivial. 
The epimorphism $R(G) \rightarrow R$ implies that $R$ is feebly nil-clean and thus Proposition 2 (iii) enables us that $R / N(R)$ is tripotent. Therefore, the main result in [6] allows us to write that $R / N(R) \cong R_{1} \times R_{2}$, where $R_{1}$ is a boolean ring and $R_{2}$ is a tripotent ring of characteristic 3 .

On the other hand, as in the proof of Proposition 1, the surjection $R \rightarrow$ $R / N(R)$ induces a surjection $R(G) \rightarrow(R / N(R))(G)$ and so in view of Lemma 2 the group $\operatorname{ring}(R / N(R))(G) \cong R_{1}(G) \times R_{2}(G)$ has to be feebly nil-clean, too. Since $2=0$ in $R_{1}$, with the aid of Lemma 1 (i) it must be that $R_{1}(G)$ is feebly nil-clean of characteristic 2 whence it is necessarily nil-clean, because under these circumstances the sum of two idempotents is again an idempotent. Employing now the quoted above result from [9], we derive that either $R_{1}$ is zero, or $R_{1}$ is non-trivial and $G=G_{2}$ is a 2-primary group.

Further, concerning the second direct factor $R_{2}$, let us assume that it is non-zero and hence a subdirect product of the field $\mathbb{Z}_{3}$. Since there exist two epimorphisms, namely $R_{2} \rightarrow \mathbb{Z}_{3}$ and $G \rightarrow G / G_{3}$, one infers that there is an induced epimorphism $R_{2}(G) \rightarrow \mathbb{Z}_{3}\left(G / G_{3}\right)$ which gives with the help of Lemma 2 that the epimorphic image $\mathbb{Z}_{3}\left(G / G_{3}\right)$ is feebly nil-clean as so is $R_{2}(G)$ being a direct factor of $(R / N(R))(G)$. According to the listed above formula of May from [8], we obtain that $\mathbb{Z}_{3}\left(G / G_{3}\right)$ is reduced and thus it is certainly tripotent by using once again Proposition 2 (iii). Consequently, the equation $z^{3}=z$ holds in the factor-group $\mathrm{G} / \mathrm{G}_{3}$, that is, $z^{2}=1$. We may have $\mathrm{G} / \mathrm{G}_{3}=\{\overline{1}\}$, that is, $\mathrm{G}=\mathrm{G}_{3}$. If now $\mathrm{G} \neq \mathrm{G}_{3}$, letting $\mathrm{g}$ be an arbitrary element in $G$, one deduces that $\left(g G_{3}\right)^{2}=G_{3}$, i.e., $g^{2} G_{3}=G_{3}$, i.e., $g^{2} \in G_{3}$. But the 3-component $\mathrm{G}_{3}$ is always 2-divisible, that is, $\mathrm{G}_{3}=\mathrm{G}_{3}^{2}$ (see, e.g., [5]). This, in turn, forces that $g=g_{3} a \subseteq G_{3} G[2]$ for some $g_{3} \in G_{3}$ and $a \in G$ [2] assuring the direct decomposition $\mathrm{G}=\mathrm{G}_{3} \times \mathrm{G}[2]$, as wanted.

"Right-to-left". Because item (1) implies at once that $R(G) \cong R$, the claim follows immediately.

We, therefore, will be concentrated on the non-trivial case for $G$, which is exactly point (2). With Proposition 1 at hand, we have that $R(G) / N(R)(G)$ is isomorphic to $(R / N(R))(G) \cong R_{1}(G) \times R_{2}(G)$ with nil $N(R)(G) \subseteq N(R(G))$. Therefore, applying Corollary 1 , one needs to show the feebly nil-cleanness of $(R / N(R))(G)$ only. To that aim, condition (a) along with the major result from $[9]$ rich us that the group ring $R_{1}(G)$ is nil-clean and so feebly nil-clean.

On the other side, concerning condition (b), the two possibilities $G=G_{3}$ and $G=G_{3} \times G[2]$ will imply that either $R_{2}(G) \cong R_{2}\left(G_{3}\right)$ or $R_{2}(G) \cong$ $R_{2}\left(G_{3}\right) \times R_{2}(G[2])$, where the validity of the latter isomorphism is formally assumed. Moreover, as the characteristic of $R_{2}$ is 3 and the equality $x^{3}=x$ holds both in $R_{2}$ and in $G[2]$, it is readily verified by utilizing only technical 
arguments that it will hold in the group ring $R_{2}(G[2])$ as well. Thus $R_{2}(G[2])$ is feebly nil-clean. We claim, besides, that $R_{2}\left(G_{3}\right)$ is also feebly nil-clean. Indeed, referring to the noticed above formula of May from [8], one detects by using routine argumentation that $\mathrm{N}\left(\mathrm{R}_{2}\left(\mathrm{G}_{3}\right)\right)=\mathrm{I}\left(\mathrm{R}_{2}\left(\mathrm{G}_{3}\right) ; \mathrm{G}_{3}\right)$. However, as noted before, $R_{2}\left(G_{3}\right) / N\left(R_{2}\left(G_{3}\right)\right)=R_{2}\left(G_{3}\right) / I\left(R_{2}\left(G_{3}\right) ; G_{3}\right) \cong R_{2}$ implies the tripotent property, which invoking Proposition 2 substantiates our claim, as expected. We, finally, just need to apply once again Lemma 1 (ii) to get the desired feebly nil-cleanness of the group ring $R_{2}(G)$, thus concluding the initial assertion for feebly nil-cleanness of the group ring $R(G)$, as promised.

As a new and somewhat more direct and comfortable confirmation that the group ring $R_{2}(G)$ is feebly nil-clean in the case when $G$ is a decomposable group as stated above, we may process like this: Since $G=G_{3} \times G$ [2], it follows at once that $R_{2}(G) \cong\left(R_{2}(G[2])\right)\left(G_{3}\right)=R_{2}^{\prime}\left(G_{3}\right)$, where we putted $R_{2}^{\prime}:=R_{2}(G[2])$. As we already showed above, $R_{2}^{\prime}$ is a ring of characteristic 3 in which the equality $x^{3}=x$ holds for all its elements. Thus, in particular, it should be reduced as well. Furthermore, as we have demonstrated, $N\left(R_{2}^{\prime}\left(G_{3}\right)\right)=I\left(R_{2}^{\prime}\left(G_{3}\right) ; G_{3}\right)$ and, consequently, $R_{2}^{\prime}\left(G_{3}\right) / N\left(R_{2}^{\prime}\left(G_{3}\right)\right)=R_{2}^{\prime}\left(G_{3}\right) / I\left(R_{2}^{\prime}\left(G_{3}\right) ; G_{3}\right) \cong R_{2}^{\prime}$ is tripotent (i.e., reduced feebly nil-clean), as expected. This gives the desired feebly nilcleanness of the group ring $R_{2}^{\prime}\left(G_{3}\right)$ which, in turn, substantiates the promised feebly nil-cleanness of $R_{2}(G)$ after all.

We close with some more comments.

Remark 1 Utilizing the stated above formula of Karpilovsky from [7], we can deduce an equivalent necessary and sufficient condition for a commutative group ring to be feebly nil-clean in terms of $\mathrm{J}(\mathrm{R})$ instead of $\mathrm{N}(\mathrm{R})$.

We end the work with a problem of interest.

Problem 1 Find a criterion when an arbitrary (not necessarily commutative) group ring is feebly nil-clean.

\section{Acknowledgement}

The present paper is partially supported by the scientific project of the Bulgarian National Science Fund under Grant KP-06 N 32/1 of Dec. 07, 2019. 


\section{References}

[1] P. V. Danchev, Feebly nil-clean unital rings, Proc. Jangjeon Math. Soc., 21 (1) (2018), 155-165.

[2] P. V. Danchev, Feebly invo-clean unital rings, Ann. Univ. Sci. Budapest (Sect. Math.), 60 (2017), 85-91.

[3] P. V. Danchev and O. Al-Mallah, UU group rings, Eurasian Bull. Math., 1 (3) (2018), 85-88.

[4] P. V. Danchev and W. Wm. McGovern, Commutative weakly nil clean unital rings, J. Algebra, 425 (5) (2015), 410-422.

[5] L. Fuchs, Abelian Groups, Springer Monographs in Math., Springer Internat. Publishing (Switzerland), 2015, 747 pp.

[6] Y. Hirano, H. Tominaga, Rings in which every element is the sum of two idempotents, Bull. Austral. Math. Soc., 37 (1988), 161-164.

[7] G. Karpilovsky, The Jacobson radical of commutative group rings, Arch. Math., 39 (1982), 428-430.

[8] W. L. May, Group algebras over finitely generated rings, J. Algebra, 39 (2) (1976), 483-511.

[9] W. Wm. McGovern, S. Raja and A. Sharp, Commutative nil clean group rings, J. Algebra Appl., 14 (6) (2015), 1550094.

[10] C. P. Milies and S. K. Sehgal, An Introduction to Group Rings, vol. 1, Springer Science and Business Media, 2002.

[11] D. Passman, The Algebraic Structure of Group Rings, Dover Publications, 2011. 